ARTIGO ORIGINAL

ORIGINAL ARTICLE

\title{
Custo de tratamento do câncer de próstata resistente à castração por meio da aplicação da técnica Delphi no Sistema de Saúde Suplementar brasileiro
}

\author{
Cost of treating castration-resistant prostate cancer by applying \\ the Delphi method to the Brazilian Private Healthcare System
}

Ligia Fernande Yoshida', Adriano Gonçalves e Silva², André Deeke Sasse², Fabio Eduardo Zola ${ }^{4,5}$, Fernando Nunes Galvão de Oliveira ${ }^{6}$, Volney Soares Lima ${ }^{7,8}$, Tiago Costa de Pádua ${ }^{9}$, Bernardo Salustio Pires ${ }^{1}$

DOI: $10.21115 / J B E S . v 11 . n 2 . p 142-52$

Palavras-chave:

câncer de próstata, resistente à castração, Sistema de Saúde Suplementar, custos, Brasil, metástase

\section{Keywords:}

prostate cancer, castrate resistance, private health system, costs, Brazil, metastasis

\section{RESUMO}

Objetivo: Estimar os custos do tratamento de pacientes com câncer de próstata não metastático resistente à castração (nmCRPC) e metastático resistente à castração (mCRPC) no Sistema de Saúde Suplementar brasileiro. Métodos: Foi realizada uma busca na literatura para avaliar os dados já disponíveis do custo do tratamento do câncer de próstata no Brasil. Como os dados são escassos, optou-se por utilizar a técnica Delphi com seis médicos oncologistas para levantamento do uso dos recursos. O painel Delphi contou com três etapas, sendo duas on-line e uma presencial, abordando recursos, frequência e porcentagem de uso de exames de imagem e laboratoriais, e dados hospitalares (consultas, pronto-socorro, internações - clínica, cirúrgica e em unidade de terapia intensiva). Também foram solicitados dados de padrões de tratamento do câncer de próstata. Por fim, dados do manejo das metástases foram requeridos. Por meio das informações coletadas, foi desenvolvido o microcusteio do câncer de próstata resistente à castração na perspectiva do Sistema de Saúde Suplementar. Resultados: O custo total de tratamento da jornada do paciente com câncer de próstata resistente à castração foi igual a 480.497,25 BRL, e o tratamento unicamente da doença não metastática foi de 189.832,79 BRL e o da doença metastática, de 290.664,46 BRL. Conclusão: Os custos do tratamento de câncer de próstata metastático são substancialmente superiores aos custos do tratamento do paciente não metastático resistente à castração de alto risco, o que parece justificar economicamente o uso de medidas que previnam ou adiem o surgimento de metástases.

\section{ABSTRACT}

Objective: To estimate the treatment costs for patients with non-metastatic castration-resistant prostate cancer (nmCRPC) and metastatic castration-resistant prostate cancer (mCRPC) within the Brazilian private healthcare system. Methods: A literature search was performed to obtain currently available data on prostate cancer treatment costs in Brazil. As national data are scarce, a Delphi method with a specialist board was chosen with six oncologists for resource estimations. The Delphi panel had three steps: two online and one in-person, addressing what resources, frequencies, and percentages of use of imaging and laboratory exams, hospital data (including medical appointments, emergency room, hospitalizations - clinical, surgical and Intensive Care Unit). Data on prostate cancer treatment patterns were also requested. Finally, metastasis management data was required. Using the collected data, we developed a micro-costing model of castration-resistant prostate cancer in

\footnotetext{
Recebido em: 02/08/2019. Aprovado para publicação em: 12/08/2019.

1. Kantar Health, São Paulo, SP, Brasil.

2. Instituto do Câncer e Transplante Curitiba, Curitiba, PR, Brasil.

3. Grupo SONHE, Oncologia, Campinas, SP, Brasil.

4. Centro de Tratamento Oncológico, Ribeirão Preto, SP, Brasil.

5. Hospital das Clínicas da Faculdade de Medicina da Universidade de São Paulo, Ribeirão Preto, SP, Brasil.

6. Clínica CLION/Grupo CAM, Salvador, BA, Brasil.

7. Oncocentro Belo Horizonte, Belo Horizonte, MG, Brasil.

8. Hospital Felício Rocho, Belo Horizonte, MG, Brasil.

9. Américas Oncologia São Paulo, São Paulo, SP, Brasil.

Informações sobre auxílios recebidos sob a forma de financiamento, equipamentos ou medicamentos: Este estudo teve suporte financeiro da Janssen-Cilag Farmacêutica Ltda. para a sua realização.

Autor correspondente: Ligia Fernande Yoshida. Edifício iTower Shopping Iguatemi, Alameda Xingu, 350, $4^{\circ}$ andar, Alphaville São Paulo, SP, Brasil. CEP: 06455-030. Telefone:+55 (11) 4133-9852. E-mail: ligia.yoshida@kantar.com
} 
Brazil. Results: The total cost for the entire castration-resistant prostate cancer patient journey was 480.497,25 BRL, with the non-metastatic disease alone costing 189.832,79 BRL and the metastatic disease costing 290.664,46 BRL. Conclusion: The costs of treating metastatic prostate cancer are substantially higher than the treatment of high-risk castration-resistant non-metastatic patients, which seems to economically justify the use of measures that prevent or postpone metastasis.

\section{Introdução}

O câncer de próstata é responsável por 14.484 mortes anualmente no Brasil, segundo dados do Instituto Nacional de Câncer (Inca), e é o segundo mais comum entre os homens no país, atrás apenas do câncer de pele não melanoma. 0 número estimado de novos casos é de 68.220 anualmente ou cerca de 61,8 a cada 100.000 homens, segundo dados de 2016 e 2017 (Inca, 2015, 2018; Brasil, 2015).

As opções terapêuticas para o câncer de próstata diferem entre os diferentes estágios da doença, assim como pelos sistemas de saúde analisados. Os pacientes com câncer de próstata resistente à castração geralmente apresentam maior risco de mortalidade, perda de qualidade de vida e pior prognóstico (NCCN, 2018; Heidenreich et al., 2014). Os pacientes com câncer de próstata não metastático de alto risco, especialmente se PSADT (PSA doubling time) $\leq 10$ meses, apresentam como opções de tratamento a terapia de privação androgênica em combinação com apalutamida ou enzalutamida (AUA, 2018; NCCN, 2018; SBOC, 2017). No caso de pacientes com câncer de próstata metastático resistente à castração, definido como progressão da doença com aumento do PSA em vigência de nível sérico de testosterona menor que $50 \mathrm{ng} / \mathrm{dL}$, as opções de tratamento apresentados pelas Diretrizes e Consenso da Sociedade Brasileira de Oncologia Clínica (SBOC) são hormonioterapia, quimioterapia ou radiofármacos (SBOC, 2017; Sasse et al., 2019).

Normalmente, o câncer de próstata tem crescimento lento e se mantém confinado à próstata - nesses casos, o câncer não causa grande impacto ao paciente. Alguns tipos de câncer de próstata, entretanto, são agressivos e podem se espalhar rapidamente (NCCN, 2018; Heidenreich et al., 2014). O aparecimento de metástases pode ocorrer para órgãos próximos, como a bexiga, ou distantes, através de vasos sanguíneos e do sistema linfático, como os ossos. Após a metástase, o câncer dificilmente é curado completamente (NCCN, 2018; Heidenreich et al., 2014).

Entre as metástases apresentadas pelos pacientes com câncer de próstata, a metástase óssea é uma das mais comuns e onerosas: aproximadamente $80 \%$ dos homens com doença avançada apresentam metástase óssea. A maioria desses pacientes experimenta dor óssea importante, comumente controlada com analgésicos opioides, radioterapia e radiofármacos, embora com resultados geralmente não satisfatórios. Além disso, pacientes que desenvolvem metástase óssea têm alto risco de apresentar complicações esqueléticas, incluindo fraturas patológicas e compressão do canal medular, resultando em morbidade significativa e alto custo hospitalar (Ferreira et al., 2004).

A quantificação do custo de tratamento do câncer é de especial interesse a tomadores de decisão na saúde, devido ao alto impacto financeiro que as neoplasias têm sobre os sistemas de saúde. Estudos sugerem que pacientes com câncer de próstata inicialmente diagnosticados com doença localizada, ao passarem para a fase metastática, apresentam aumento substancial dos custos e recursos médicos utilizados. Um estudo de coorte retrospectivo avaliou os custos médicos 12 meses antes da ocorrência da metástase e até 12 meses depois da metástase. Entre os pacientes que não apresentaram metástase, esse custo foi de 2.746 USD por paciente por mês. Esses custos aumentaram para 4.767 USD [intervalo de confiança (IC) de 95\%: 4.623 USD a 4.910 USD] um mês antes do diagnóstico de metástase, permanecendo superiores ao custo do paciente sem metástase (4.677 USD em 12 meses; IC de 95\%: 4.549 USD a 4.805 USD) (Li et al., 2017).

Um estudo brasileiro avaliou o custo de pacientes com câncer de próstata com metástase óssea no Sistema de Saúde Suplementar. O estudo utilizou a base administrativa da Orizon com um horizonte temporal de 181 dias. Foram registrados os dados de entradas hospitalares ou ambulatoriais de 181 pacientes. Os resultados mostraram que o custo médio de um paciente é de 118.184 BRL. Asano et al. concluíram que o câncer de próstata com metástase óssea está associado a um considerável impacto econômico, uma vez que o paciente nessa condição necessita, por exemplo, de maior quantidade e permanência de hospitalizações (Asano et al., 2018). No entanto, dados na literatura sobre o custo do câncer de próstata no Sistema de Saúde Suplementar ainda são escassos. Uma das formas de coletar dados detalhados de quantidade e custo de recursos utilizados para a estimativa de custo de tratamentos é a metodologia Delphi. Esse método é uma fonte poderosa de investigação, uma vez que agrupa a opinião de diferentes especialistas, resultando em consenso de uma temática muitas vezes abrangente e complexa. Uma das principais vantagens é a contribuição de vários especialistas ao invés de um único indivíduo, tornando o processo mais válido do que a opinião de um único especialista (Marques \& Freitas, 2018).

A técnica Delphi original envolve quatro rodadas com especialistas: uma rodada de questionário focado em respostas qualitativas, uma rodada de questionário focado em respostas quantitativas e mais duas rodadas baseadas nas respostas anteriores. Versões modificadas da metodologia podem se utilizar de painéis presenciais e pesquisa da literatura em 
parte das rodadas (Thangaratinam \& Redman, 2005). Por não ser um estudo de base estatística, a metodologia Delphi não necessita de participantes representativos da população geral, mas sim de especialistas no assunto que, idealmente, cooperem e contribuam com ideias. Desse modo, a seleção dos participantes é o fator primário para um estudo Delphi (Gordon, 1994). Assim como outras metodologias que investigam questões complexas a partir da experiência e opinião de um grupo, uma das desvantagens desse método está relacionada aos especialistas que podem optar por simplesmente seguir a resposta do grupo ou então a manipular os resultados (Marques \& Freitas, 2018). Apesar disso, o uso desse método é considerado confiável na obtenção de consenso entre especialistas no planejamento e previsão de áreas específicas e uma técnica válida no âmbito da economia da saúde, sendo citada como opção de fonte de dados pelas Diretrizes Metodológicas de Análise de Impacto Orçamentário (Brasil, 2014).

Considerando a limitação de dados nacionais em relação ao custo do tratamento do câncer de próstata no país, o objetivo deste estudo é realizar um microcusteio do câncer de próstata resistente à castração utilizando a técnica Delphi sob a perspectiva do Sistema de Saúde Suplementar brasileiro.

\section{Métodos}

Este estudo avaliou os custos diretos do tratamento de pacientes com câncer de próstata nas seguintes fases da doença: câncer de próstata não metastático resistente à castração (non-metastatic castration-resistant prostate cancer - nmCRPC) de alto risco e câncer de próstata metastático resistente à castração (metastatic castration-resistant prostate cancer $\mathrm{mCRPC}$ ) na terapia de primeira (1L), segunda (2L), terceira (3L) e quarta ou subsequentes linhas (4L+). O desenvolvimento desse microcusteio seguiu as orientações da proposta metodológica de microcusteio para o Brasil (Etges et al., 2019).

Uma busca na literatura por dados dos custos do câncer de próstata no Brasil foi realizada com o objetivo de avaliar dados já disponíveis. A busca foi feita nas bases de dados Medlars Online (MEDLINE), Biblioteca Virtual em Saúde (BVS) e International Society for Pharmacoeconomics and Outcomes Research (ISPOR) Scientific Presentations Database. Não foram encontradas informações específicas do custo do câncer de próstata do paciente com nmCRPC e mCRPC no Brasil, sendo então adotado o método Delphi com especialistas para realizar o levantamento dos recursos utilizados em cada uma dessas fases da doença e, assim, desenvolver o microcusteio.

\section{Painel Delphi}

Foram realizadas três etapas para o painel Delphi: nas duas primeiras, os participantes responderam ao questionário on-line e, na última etapa, o questionário foi respondido presencialmente. Foi elaborado um questionário com perguntas referentes ao uso dos recursos para o diagnóstico e tratamento das diferentes fases do nmCRPC e mCRPC da $1 \mathrm{~L}$ até a $4 \mathrm{~L}+$ de tratamento. Os seguintes grupos foram abordados: exames, englobando os exames laboratoriais e de imagem; recursos hospitalares, incluindo consulta, atendimento no pronto-socorro e internações (clínica, cirúrgica e na unidade de terapia intensiva - UTI) devidos ao câncer de próstata. O último grupo abordado foi o dos medicamentos, sendo assim elaboradas questões referentes ao padrão de tratamento de acordo com suas práticas clínicas. No caso do paciente metastático, questionou-se quais eram os principais sítios de metástase, a porcentagem de pacientes com metástase e qual o manejo para o tratamento. Além de quais recursos são utilizados, a porcentagem de uso e a frequência de utilização também foram solicitadas para os exames, drogas e custos hospitalares.

Na primeira etapa, foram enviadas cerca de cem questões aos participantes. As questões foram criadas com base em diretrizes e no consenso de tratamento do câncer de próstata da SBOC (SBOC, 2017; Sasse et al., 2019). Os recursos abordados pelo questionário on-line relacionados a exames, custos hospitalares e medicamentos estão descritos na Tabela 1; os que estão declarados como "não solicitado na prática clínica" são aqueles que não foram citados como parte da rotina de tratamento dos pacientes pelos especialistas durante $\mathrm{O}$ painel Delphi. Em seguida, as respostas foram compiladas e analisadas para verificar seu padrão. Na segunda etapa, foi enviado um novo questionário com as mesmas perguntas do primeiro; no entanto, dessa vez foi dada visibilidade do padrão de respostas da etapa anterior aos participantes, porém mantendo-se o anonimato, o objetivo foi avaliar se, baseados nessa informação, eles chegariam mais próximo a um consenso, conforme a metodologia do painel Delphi (Thangaratinam \& Redman, 2005).

Após a segunda etapa on-line, as respostas foram novamente compiladas e analisadas. Na terceira etapa, presencial, o moderador apresentou as respostas da segunda etapa de forma anônima e perguntou novamente as mesmas perguntas dos questionários prévios na intenção de que os participantes discutissem o tema e chegassem mais próximo a um consenso. As perguntas que chegaram a um consenso com todos os participantes na primeira e na segunda etapa não foram novamente questionadas na terceira etapa, uma vez que as respostas das etapas anteriores já apresentavam um consenso do grupo atingido. A Figura 1 mostra o fluxograma da metodologia utilizada no painel Delphi.

O painel Delphi contou com seis médicos oncologistas que possuem especialização na área geniturinária, especialmente no tratamento de pacientes com câncer de próstata, e que atuam no Sistema de Saúde Suplementar.

\section{Microcusteio}

Com base nas informações coletadas no painel Delphi, foi realizado um modelo de microcusteio do nmCRPC e mCRPC da $1 \mathrm{~L}$ até $4 \mathrm{~L}+$. Os custos dos medicamentos foram retirados da lista de preço máximo regulado pela Câmara de Regu- 
Tabela 1. Exames laboratoriais, exames de imagem, medicamentos e recursos hospitalares considerados para o questionário on-line

\begin{tabular}{|c|c|c|}
\hline Recurso & Custos unitários & Referência \\
\hline \multicolumn{3}{|l|}{ Exames } \\
\hline Hemograma & 71,27 BRL & AMB 2016 \\
\hline Ureia & $61,92 \mathrm{BRL}$ & AMB 2016 \\
\hline TGO & $68,36 \mathrm{BRL}$ & AMB 2016 \\
\hline TGP & $64,70 \mathrm{BRL}$ & AMB 2016 \\
\hline Bilirrubina & $64,70 \mathrm{BRL}$ & AMB 2016 \\
\hline Albumina & $64,70 \mathrm{BRL}$ & AMB 2016 \\
\hline Fosfatase alcalina & $61,92 \mathrm{BRL}$ & AMB 2016 \\
\hline Coagulograma & $68,36 \mathrm{BRL}$ & AMB 2016 \\
\hline PSA & $59,13 \mathrm{BRL}$ & AMB 2016 \\
\hline Testosterona & $113,08 \mathrm{BRL}$ & AMB 2016 \\
\hline Cálcio iônico & $75,33 \mathrm{BRL}$ & AMB 2016 \\
\hline $\mathrm{DHL}$ & $68,36 \mathrm{BRL}$ & AMB 2016 \\
\hline \multicolumn{3}{|l|}{ Exames de imagem } \\
\hline Cintilografia óssea & $393,20 \mathrm{BRL}$ & AMB 2016 \\
\hline $\begin{array}{l}\text { Tomografia computadorizada do abdome, } \\
\text { pelve e tórax }\end{array}$ & Não solicitado na prática clínica & - \\
\hline Ressonância magnética do abdome, pelve e tórax & $682,75 \mathrm{BRL}$ & AMB 2016 \\
\hline $\begin{array}{l}\text { Ressonância magnética de corpo inteiro com } \\
\text { protocolo de difusão }\end{array}$ & Não solicitado na prática clínica & - \\
\hline $\begin{array}{l}\text { Tomografia computadorizada direcionada aos } \\
\text { sintomas do paciente }\end{array}$ & Não solicitado na prática clínica & - \\
\hline Ressonância magnética de coluna & $1.218,00 \mathrm{BRL}$ & AMB 2016 \\
\hline PET/CT & Não solicitado na prática clínica & - \\
\hline PET-CT PSMA & Out-of-pocket & - \\
\hline \multicolumn{3}{|l|}{ Medicamentos - Custo de tratamento } \\
\hline Apalutamida + ADT & $12.095,18 \mathrm{BRL}$ & Média das apresentações - CMED junho, 2019 \\
\hline Enzalutamida + ADT & $12.095,19 \mathrm{BRL}$ & Média das apresentações - CMED junho, 2019 \\
\hline Bicalutamida + ADT & $1.856,47 \mathrm{BRL}$ & Média das apresentações - CMED junho, 2019 \\
\hline Gosserrelina & $440,20 \mathrm{BRL}$ & Média das apresentações - CMED junho, 2019 \\
\hline Leuprolide & $121,79 \mathrm{BRL}$ & Média das apresentações - CMED junho, 2019 \\
\hline Triptorrelina & $329,31 \mathrm{BRL}$ & Média das apresentações - CMED junho, 2019 \\
\hline Docetaxel & 7.006,71 BRL & Média das apresentações - CMED junho, 2019 \\
\hline Cabazitaxel & 15.191,01 BRL & Média das apresentações - CMED junho, 2019 \\
\hline Mitoxantrona & Não solicitado na prática clínica & - \\
\hline Ciclofosfamida & Não solicitado na prática clínica & - \\
\hline Radium-223 & $20.125,95 \mathrm{BRL}$ & Média das apresentações - CMED junho, 2019 \\
\hline Samário e estrôncio & Não solicitado na prática clínica & - \\
\hline Abiraterona & 8.722,13 BRL & Média das apresentações - CMED junho, 2019 \\
\hline Cetoconazol & Não solicitado na prática clínica & - \\
\hline \multicolumn{3}{|l|}{ Recursos Hospitalares } \\
\hline Diária de internação em UTI & 4.023,11 BRL & \\
\hline Diária de internação na clínica cirúrgica & $1.882,12 \mathrm{BRL}$ & \\
\hline Diária de internação na clínica médica & $1.882,12 \mathrm{BRL}$ & \\
\hline Consulta & $91,65 \mathrm{BRL}$ & \\
\hline Pronto-socorro & $91,65 \mathrm{BRL}$ & \\
\hline
\end{tabular}




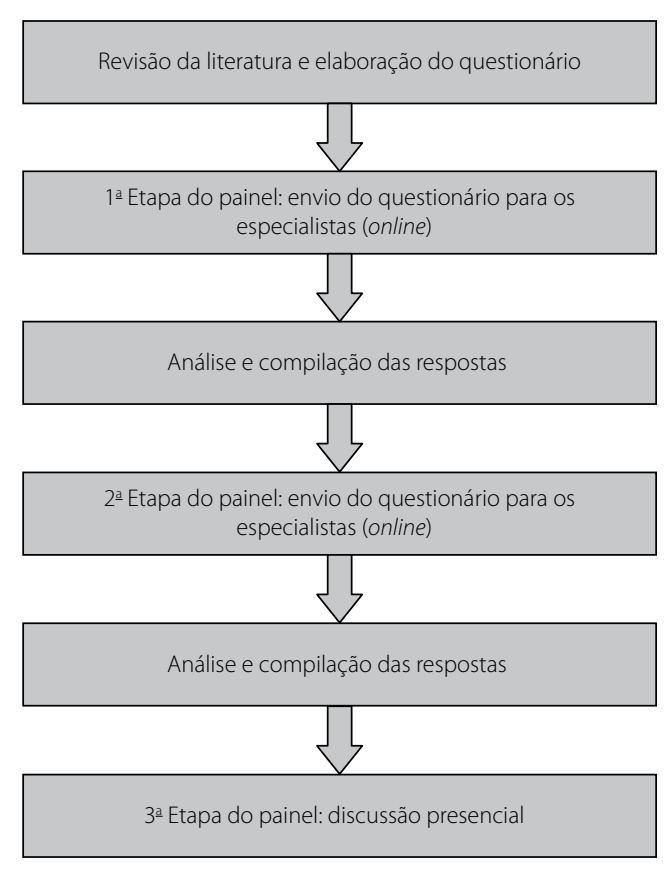

Figura 1. Fluxograma do painel Delphi.

lação do Mercado de Medicamentos (CMED). Foram utilizados os preços de fábrica acrescidos de alíquota de Imposto sobre Circulação de Mercadorias e Serviços (ICMS) de 18\% (porcentagem aplicável aos estados AM, AP, BA, CE, MA, MG, $P B, P E, P I, P R, R N, R S, S E, S P, T O$ e RJ) e consultados em junho de 2019. O preço dos medicamentos foi calculado com base na média das apresentações com o mesmo princípio ativo. Os custos de materiais de uso hospitalar foram retirados da Simpro de julho de 2019 e os honorários médicos e procedimentos, da Classificação Brasileira Hierarquizada de Procedimentos Médicos (CBHPM) de 2016 (AMB, 2016). A quantidade de materiais, medicamentos e honorários médicos necessários em cada cirurgia ou internação foi estimada por uma enfermeira auditora especialista com experiência no Sistema de Saúde Suplementar brasileiro. Os custos foram apresentados em reais (BRL).

A técnica utilizada foi a de bottom-up (baixo para cima), ou seja, foram coletados os custos unitários dos recursos, porcentagem e frequência de uso para chegar ao custo individual do paciente. Os dados utilizados foram majoritariamente baseados nas informações coletadas durante o painel Delphi, com alguns dados baseados em opinião individual de especialista, como a porcentagem de distribuição de uso da metadona, buprenorfina e morfina e a porcentagem de uso da ressecção transuretral de próstata entre pacientes metastáticos e não metastáticos, uma vez que foram tópicos adicionais que surgiram como comentários durante o painel. Em momentos nos quais não houve consenso dos participantes, foi coletada a opinião individual de todos os seis par- ticipantes e realizada uma média das respostas. Caso fosse necessário arredondar o valor médio, por exemplo, 7,5 dias de internação, sempre se optou por arredondar para cima (para o valor mais conservador), com o intuito de evitar subestimar os custos do tratamento.

\section{Tempo de tratamento}

Durante o painel, foram questionados para cada linha de tratamento (de nmCRPC até o mCRPC 4L+): o percentual de pacientes que utilizam hormonioterapia de primeira e segunda geração, quimioterapia e radiofármaco, e o tempo médio de cada tratamento, uma vez que dados de mundo real no país com essa informação são escassos. Para a obtenção do tempo de tratamento utilizado nos cálculos para as linhas avaliadas, foi feita uma média ponderada do tempo de cada tratamento (hormonioterapia de primeira e segunda geração, quimioterapia e radiofármaco) pelo seu percentual de uso.

\section{Análise de sensibilidade}

Foi realizada uma análise de sensibilidade determinística univariada para avaliar a influência da incerteza e a variabilidade dos parâmetros utilizados nos resultados do microcusteio. Os valores máximos e mínimos utilizados se basearam nos dados informados durante o próprio painel Delphi, por exemplo, variação dos dias de internação. Para parâmetros nos quais não se aplica essa variação, foi utilizada uma variação de $\pm 10 \%$ do valor-padrão (Tabela 2).

\section{Resultados}

O padrão de tratamento e uso de recursos no tratamento do paciente diagnosticado com câncer de próstata resistente à castração apresentou pequena variação por especialista/instituição, e os resultados mostraram uma tendência de maior utilização de recursos em pacientes em linhas mais tardias. Foi possível observar um aumento na frequência de recursos hospitalares, como consultas, internações e idas ao pronto-socorro, em pacientes metastáticos em relação aos não metastáticos. O mesmo foi observado na realização de exames de acompanhamento, laboratoriais ou de imagem. A porcentagem de uso dos recursos hospitalares e o número de diárias em cada uma das linhas de tratamento são descritos na Tabela 3. As porcentagens de pacientes que utilizam os exames laboratoriais e de imagem em cada linha de tratamento estão apresentadas nas Tabelas 4 e 5, respectivamente.

\section{Padrão de tratamento}

O padrão de tratamento discutido pelo grupo apresentou poucas alterações em relação à conduta de tratamento nos diferentes cenários da doença. Foi estimado que 100\% dos pacientes, tanto na fase de nmCRPC quanto na de MCRPC $1 \mathrm{~L}$, são tratados por uma linha de tratamento; 90\% em mCRPC 2L, 65\% em mCRPC $3 \mathrm{~L}$ e, por último, 45\% em mCRPC recebem uma $4 \mathrm{~L}$ de tratamento. Na Tabela 6 constam os resultados do padrão de tratamento. 
Tabela 2. Parâmetros da análise de sensibilidade e variações considerando os 16 parâmetros mais sensíveis

\begin{tabular}{lccc}
\hline Parâmetro & Valor utilizado & Valor mínimo & Valor máximo \\
\hline Custo de tratamento com inibidor seletivo de androgênico (abiraterona) & $8.722,13$ & $7.849,92$ & $9.594,35$ \\
\hline Custo de tratamento com ADT & 891,30 & 802,17 & 980,43 \\
\hline Custo de tratamento com cabazitaxel & $15.191,01$ & $13.671,91$ & $16.710,12$ \\
\hline Custo de tratamento com docetaxel & $7.006,71$ & $6.306,04$ & $7.707,38$ \\
\hline Custo de tratamento com hormonioterapia antiandrogênica de 2a geração & $12.095,18$ & $10.885,67$ & $13.304,71$ \\
\hline Custo de tratamento com Radium - 223 & $20.125,95$ & $18.113,36$ & $22.138,55$ \\
\hline Porcentagem de pacientes com metástases & 0,35 & 0,32 & 0,39 \\
\hline Progressão para mCRPC 2L & 0,90 & 0,81 \\
\hline Progressão para mCRPC 3L & 0,65 & 0,59 \\
\hline Progressão para mCRPC 4L+ & 0,45 & 0,41 \\
\hline Tempo em quimioterapia mCRPC 3L & 4,00 & 3,60 \\
\hline Tempo em hormonioterapia antiandrogênica de 2a geração nmCRPC & 24,00 & 21,60 \\
\hline Tempo em internação clínica mCRPC 2L & 5,00 & 3,00 \\
\hline Tempo em internação clínica mCRPC 3L & 8,00 & 4,40 \\
\hline Tempo em internação clínica mCRPC 4L+ & 8,00 & $1.882,12$ & $1.693,91$ \\
\hline Valor diário de internação em clínica médica & 5,00 \\
\hline
\end{tabular}

Tabela 3. Recursos hospitalares - diárias e porcentagem de utilização

\begin{tabular}{|c|c|c|c|c|c|c|c|c|c|c|}
\hline Recurso & nmCRPC & Diárias & $\begin{array}{l}\text { \% de uso } \\
\text { mCRPC 1L }\end{array}$ & Diárias & $\begin{array}{l}\% \text { de uso } \\
\text { mCRPC } 2 L\end{array}$ & Diárias & $\begin{array}{l}\text { \% de uso } \\
\text { mCRPC } 3 \mathrm{~L}\end{array}$ & Diárias & $\begin{array}{c}\% \text { de uso } \\
\text { mCRPC 4L+ }\end{array}$ & Diárias \\
\hline Consulta ambulatorial & $100 \%$ & - & $100 \%$ & - & $100 \%$ & - & $100 \%$ & - & $100 \%$ & - \\
\hline Pronto-socorro & $20 \%$ & - & $40 \%$ & - & $50 \%$ & - & $83 \%$ & - & $80 \%$ & - \\
\hline Internação clínica & $1 \%$ & 3 & $5 \%$ & 5 & $25 \%$ & 5 & $50 \%$ & 8 & $100 \%$ & 8 \\
\hline Clínica Cirúrgica & $10 \%$ & 3 & $10 \%$ & 8 & $10 \%$ & 8 & $10 \%$ & 8 & $10 \%$ & 10 \\
\hline Internação na UTI & $0 \%$ & - & $2 \%$ & 2 & $5 \%$ & 3 & $0 \%$ & 2 & $0 \%$ & - \\
\hline
\end{tabular}

Tabela 4. Exames laboratoriais de seguimento - frequência e porcentagem de uso

\begin{tabular}{lcc}
\hline Nome do exame & $\begin{array}{c}\text { Porcentagem } \\
\text { de uso }\end{array}$ & $\begin{array}{c}\text { Frequência de } \\
\text { solicitação }\end{array}$ \\
\hline $\begin{array}{l}\text { Câncer de próstata não } \\
\text { metastático resistente à } \\
\text { castração de alto risco }\end{array}$ & & \\
\hline PSA & $100 \%$ & Trimestral \\
\hline Testosterona & $100 \%$ & Trimestral \\
\hline $\begin{array}{l}\text { Câncer de próstata } \\
\text { metastático resistente } \\
\text { castração 1L, 2L, 3L ou 4L+ }\end{array}$ & & \\
\hline PSA & $100 \%$ & Mensal \\
\hline Testosterona & $100 \%$ & Mensal \\
\hline
\end{tabular}

\section{Metástase}

Durante o painel, foi reportado pelos médicos que em torno de 35\% dos pacientes desenvolvem metástase. Desses pacientes, cerca de 90\% apresentarão metástase óssea, cujo tra- tamento inclui opioides para manejo da dor, como buprenorfina, metadona e morfina, com porcentagem de uso de 30\%, $30 \%$ e 40\%, respectivamente. Dos pacientes com metástase óssea, 30\% sofrem fraturas e, desses, apenas 10\% seriam elegíveis para a cirurgia. A cirurgia de coluna foi apontada como uma das mais comuns em caso de fraturas, enquanto a necessidade de descompressão medular foi estimada em 15\% dos casos de metástase óssea. Outro tipo de tratamento específico citado foi para a metástase leptomeníngea (em cerca de $7 \%$ dos pacientes), na qual é utilizada a radioterapia em aproximadamente 20\% dos casos. Foi ainda indicado o uso de denosumabe em cerca de 55\% dos pacientes com metástase óssea e do ácido zoledrônico para o restante.

\section{Cirurgias}

Foram apontadas durante o painel e consideradas na análise as seguintes cirurgias: duplo J em 5\% dos pacientes no cenário $\mathrm{nmCPRC}$ e ressecção transuretral de próstata em $9 \%$ e 10\% dos pacientes mCPRC e nmCPRC, respectivamente. 


\section{Custos}

Considerando o padrão de uso de recurso e frequência, o custo total do tratamento do paciente com câncer de próstata resistente à castração foi de 480.497,25 BRL. Na estratificação pela existência ou não de metástase, foi encontrado o valor de $\mathrm{R} \$ 189.832,79$ para o tratamento do paciente nmCRPC, enquanto no mCRPC o valor total foi de $290.664,46$ BRL. O custo por linha de tratamento do paciente MCRPC foi de 132.841,82 BRL na 1L, 54.437,47 BRL na 2L, 57.405,25 BRL na 3L e 34.810,29 BRL na 4L+. Esses dados foram calculados levando-se em conta a porcentagem de pacientes em tratamento em cada fase da doença. Os custos totais com cirurgias e/ou manejo de metástases, considerando a porcentagem de utilização, foram de 5.461,10 BRL na fase nmCRPC e de 11.169,63 BRL na fase metastática. A descrição dos custos divididos por tipo de recurso e com base na porcentagem de pacientes tratando em cada linha e também o tempo médio de tratamento está descrita na Tabela 7.

O custo da cirurgia de coluna foi estimado em 47.916,32 BRL, incluindo honorários médicos, materiais e medicamentos. A descompressão medular, necessária em cerca de 15\% dos casos de metástase óssea, apresenta custo associado de $57.179,54 \mathrm{BRL}$. O custo de tratamento com denosumabe foi de BRL 735,26 e de BRL 667,68 com o ácido zoledrônico. Considerando os percentuais informados de utilização de denosumabe ou ácido zoledrônico, obteve-se um

Tabela 5. Exames de imagem de seguimento - frequência e porcentagem de uso

\begin{tabular}{lccc}
\hline Nome do exame & Cintilografia óssea & $\begin{array}{c}\text { Tomografia do tórax, } \\
\text { abdome e pelve }\end{array}$ & Ressonância da coluna \\
\hline Porcentagem de uso para nmCRPC & $100 \%$ & $100 \%$ & Não solicitado \\
\hline Frequência de solicitação para nmCRPC & Semestral & Semestral & $10 \%$ \\
\hline Porcentagem de uso para mCRPC 1L & $100 \%$ & $50 \%$ & $1 \mathrm{vez}$ \\
\hline Frequência de solicitação para mCRPC 1L & Semestral & Semestral & $25 \%$ \\
\hline Porcentagem de uso para mCRPC 2L & $100 \%$ & $50 \%$ & $1 \mathrm{vez}$ \\
\hline Frequência de solicitação para mCRPC 2L & Semestral & $100 \%$ & $67 \%$ \\
\hline Porcentagem de uso para mCRPC 3L & $100 \%$ & Trimestral & $1 \mathrm{vez}$ \\
\hline Frequência de solicitação para mCRPC 3L & Trimestral & $100 \%$ & $100 \%$ \\
\hline Porcentagem de uso para mCRPC 4L+ & $100 \%$ & Trimestral & $1 \mathrm{vez}$ \\
\hline Frequência de solicitação para mCRPC 4L+ & Trimestral & & \\
\hline
\end{tabular}

Tabela 6. Porcentagem de uso de cada terapia

\begin{tabular}{|c|c|c|c|c|c|}
\hline Terapia & $\begin{array}{c}\text { \% de uso para } \\
\text { nmCRPC }\end{array}$ & $\begin{array}{c}\text { \% de uso para } \\
\text { mCRPC 1L }\end{array}$ & $\begin{array}{c}\text { \% de uso para } \\
\text { mCRPC 2L }\end{array}$ & $\begin{array}{c}\text { \% de uso para } \\
\text { mCRPC 3L }\end{array}$ & $\begin{array}{c}\text { \% de uso para } \\
\text { mCRPC 4L+ }\end{array}$ \\
\hline Hormonioterapia de $2^{a}$ geração & $58 \%$ & $90 \%$ & $20 \%$ & $58 \%$ & $39 \%$ \\
\hline Hormonioterapia de 1 a geração & $7 \%$ & $0 \%$ & $0 \%$ & $0 \%$ & $0 \%$ \\
\hline Quimioterapia & $0 \%$ & $10 \%$ & $70 \%$ & $28 \%$ & $50 \%$ \\
\hline Radiofármaco & $0 \%$ & $0 \%$ & $10 \%$ & $14 \%$ & $11 \%$ \\
\hline ADT & $30 \%$ & - & - & - & - \\
\hline Vigilância ativa & $5 \%$ & $0 \%$ & $0 \%$ & $0 \%$ & $0 \%$ \\
\hline
\end{tabular}

Tabela 7. Custo global em cada linha de tratamento e por categoria

\begin{tabular}{|c|c|c|c|c|c|c|}
\hline Tipo de custo & $\mathrm{nmCRPC}$ & mCRPC 1L & mCRPC 2L & mCRPC 3L & mCRPC 4L+ & $\begin{array}{l}\text { Total por } \\
\text { categoria }\end{array}$ \\
\hline Hospitalares & $R \$ 1.863,94$ & $\mathrm{R} \$ 3.229,81$ & $\mathrm{R} \$ 4.732,42$ & $R \$ 6.274,26$ & $R \$ 7.805,66$ & $R \$ 23.906,10$ \\
\hline Medicamentos & $\mathrm{R} \$ 175.921,30$ & $\mathrm{R} \$ 124.062,59$ & $R \$ 45.912,46$ & $\mathrm{R} \$ 48.078,53$ & $R \$ 24.876,86$ & $\mathrm{R} \$ 418.851,74$ \\
\hline Exames & $R \$ 6.586,44$ & $R \$ 5.549,42$ & $R \$ 3.792,59$ & $R \$ 3.052,46$ & $R \$ 2.127,76$ & $R \$ 21.108,68$ \\
\hline Total por linha de tratamento & $\mathrm{R} \$ 184.371,69$ & $R \$ 132.841,82$ & $\mathrm{R} \$ 54.437,47$ & $R \$ 57.405,25$ & $\mathrm{R} \$ 34.810,29$ & - \\
\hline Cirurgias e/ou manejo de metástases & $\mathrm{R} \$ 5.461,10$ & \multicolumn{4}{|c|}{$\mathrm{R} \$ 11.169,63$} & - \\
\hline Total por nmCRPC e mCRPCo & $\mathrm{R} \$ 189.832,79$ & \multicolumn{5}{|c|}{$R \$ 290.664,46$} \\
\hline
\end{tabular}


custo total de 127,38 BRL e de 94,64 BRL por paciente, respectivamente. Outro tipo de tratamento específico foi para a metástase leptomeníngea, no qual é utilizada a radioterapia com valor unitário de 15.900,00 BRL. Quanto ao custo relacionado ao manejo da dor, o valor médio foi de 676,05 BRL por 60 dias de tratamento com opioides.

Em relação às demais cirurgias apontadas durante o painel, a ressecção transuretral de próstata apresentou custo total de 37.033,84 BRL; implantação do port-a-cath em caso de infusão de drogas injetáveis, custando 10.154,33 BRL; orquiectomia, de 5.997,77 BRL e duplo J, de 35.154.39 BRL.

O custo mensal do tratamento de nmCRPC, somando os medicamentos, exames e recursos hospitalares, foi igual a 9.311,70 BRL. No tratamento do paciente mCRPC, tais custos mensais de tratamento de MCRPC por paciente foram iguais a 11.210,28 BRL para a 1L, 7.457,19 BRL para a 2L, 11.153,87 BRL para a 3L, e 11.603,43 BRL para a 4L+. Os custos mensais com medicamentos, exames e gastos hospitalares estão descritos na Figura 2. Conforme o cálculo do tempo médio de tratamento, foram considerados 20, 12, 7, 5 e 3 meses nas linhas de tratamento nmCRPC, mCRPC 1L, mCRPC 2L, mCRPC $3 \mathrm{~L}$ e mCRPC 4L+, respectivamente.

\section{Análise de sensibilidade}

A análise de sensibilidade evidenciou que os parâmetros mais sensíveis foram o custo de tratamento com hormonioterapia antiandrogênica de segunda geração e o tempo médio de tratamento com hormonioterapia de segunda geração do nmCRPC (Figura 3). O custo do tratamento do câncer de próstata resistente à castração variou entre 455 mil BRL e 505 mil BRL. Em uma análise de cenários com base nesses parâmetros, caso o custo mínimo do tratamento com hormonioterapia antiandrogênica de segunda geração fosse de 10.885,67 $\mathrm{BRL}$, o custo de tratamento do câncer de próstata seria de 455.586,50 BRL, mas se o custo máximo fosse de 13.304,71 BRL, o custo do câncer de próstata seria de 505.408,00 BRL. Para o segundo parâmetro mais sensível caso o tempo de tratamento com hormonioterapia antiandrogênica de segunda geração para nmCRPC em vez de 24 meses, como apontado pelos especialistas, fosse de 26,4 meses, o custo de tratamento seria de $499.048,05$ BRL. Se esse tempo variasse para 21,6 meses, o custo de tratamento seria de 463.022,40 BRL.

\section{Discussão}

Os resultados da avaliação do uso de recursos mostraram que o câncer de próstata resistente à castração no país está relacionado a uma maior utilização de recursos hospitalares e de medicamentos em pacientes que apresentam metástase, sendo o custo de um paciente sem metástase potencialmente inferior ao custo de um paciente metastático. $O$ custo de um paciente com nmCRPC correspondeu a 40\% dos custos totais do tratamento do câncer de próstata resistente à castração, se comparado a $60 \%$ de um paciente com mCRPC.
Custos hospitalares mensal por paciente no tratamento do câncer de próstata

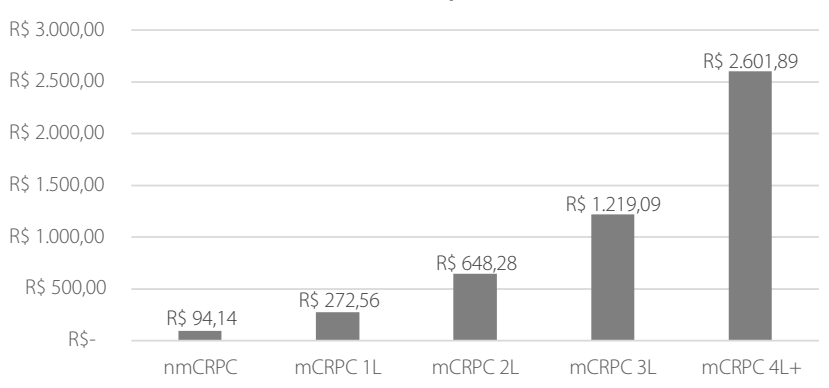

Custos com medicamentos mensal por paciente no tratamento do câncer de próstata

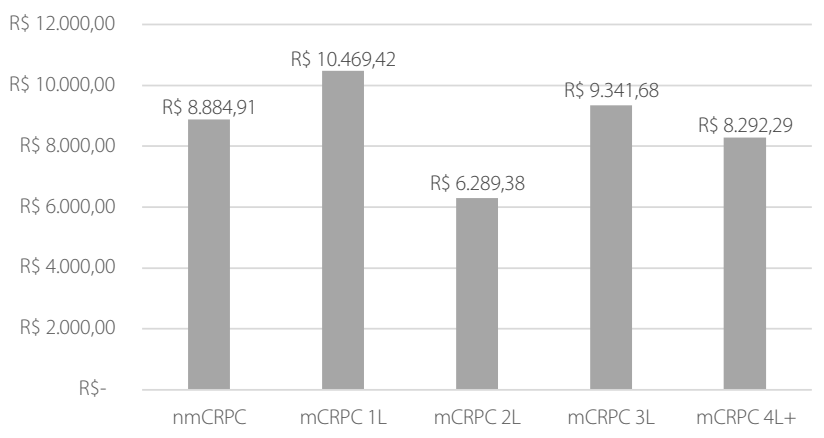

Custos com exames mensal por paciente no tratamento do câncer de próstata

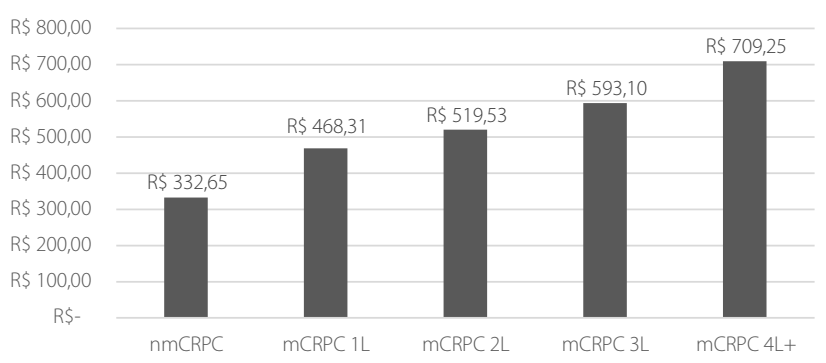

Figura 2. Custo mensal com gastos hospitalares, medicamentos e exames por paciente.

Os principais custos se referem aos medicamentos, ou seja, o custo de aquisição do medicamento, infusão no caso dos injetáveis, honorários médicos e taxas, seguidos dos custos hospitalares, que englobam as consultas médicas, pronto-socorro, cirurgia e internação (clínica, cirúrgica e UTI), e, por fim, aos exames de imagem e laboratoriais.

Sem considerar custos com manejo de metástase ou demais cirurgias, a soma dos tratamentos da MCRPC $1 \mathrm{~L}$ e mCRPC 2 L é de cerca de 187.279,29 BRL, valor superior ao custo do tratamento do nmCRPC de 184.371,69 BRL para praticamente o mesmo tempo de tratamento. Os principais custos que influenciam esse resultado são os custos dos medicamentos: de 124.062,59 BRL em mCRPC 1L e de 45.912,46 BRL em mCRPC 2L, 48.078,53 BRL em mCRPC 3L, 24.876,86 $\mathrm{BRL}$ em mCRPC 4L, totalizando, apenas nessa categoria, 242.930,44 BRL (vs. 175.921 BRL em nmCRPC). Além disso, es- 
tamos considerando o custo do tratamento com a taxa de pacientes que chegam em cada linha de terapia, sendo no cenário não metastático e mCRPC $1 \mathrm{~L}$ de 100\% de pacientes tratados, no mCRPC $2 \mathrm{~L}$ de $90 \%$, na $3 \mathrm{~L}$ de $65 \%$ e na $4 \mathrm{~L}+$ de 45\%. Ainda assim, mesmo com uma menor porcentagem de pacientes passando para linhas subsequentes de tratamento na fase metastática, o custo do paciente metastático é superior ao de um paciente não metastático. Vale ressaltar que o paciente com nmCRPC nesse contexto já é tratado com hormonioterápicos de última geração (58\% dos pacientes tratados com hormonioterapia antiandrogênica de segunda geração), enquanto na fase metastática a quimioterapia apresenta custo muito menor e alta porcentagem de utilização, chegando a 70\% no mCRPC 2L, por exemplo. Isso reforça a efetividade dos tratamentos hormonioterápicos de última geração no cenário do nmCRPC, que conseguem reproduzir um benefício superior para o paciente a um custo menor que as demais terapias no cenário metastático.

O custo mensal do tratamento do câncer de próstata resistente à castração aumenta do paciente com nmCRPC até o paciente com mCRPC 4L+ em relação aos gastos hospitalares iniciando com custo de 94,14 BRL até 2.601,89 BRL para o paciente MCRPC 4L+. Para a categoria de exames, o custo inicia com 332,65 BRL dos pacientes nmCRPC até 709,25 BRL - logo os custos relacionados a exames e recursos hospitalares são crescentes ao longo das linhas de tratamento. Na categoria medicamentos, os custos mensais iniciam com 8.884,91 BRL em pacientes nmCRPC, variando de 10.469,42 BRL em mCPRC $1 \mathrm{~L}$ e 6.289,38 BRL em mCRPC 2L+.

Os custos hospitalares aumentaram ao longo das linhas de tratamento do nmCRPC até mCRPC 4L+, uma vez que a porcentagem de pacientes que utilizam, por exemplo, internação clínica variaram de 1\% no nmCRPC até 100\% dos pacientes sendo internados pelo menos uma vez no mCRPC $4 \mathrm{~L}+$. Da mesma forma, o pronto-socorro iniciou com cerca de 20\% dos pacientes com nmCRPC necessitando desse recurso e atingindo até $80 \%$ dos pacientes com mCRPC $4 L+$. O recurso que mais difere desse padrão de aumento ao longo das linhas de tratamento é a utilização da UTI, na qual os especialistas apontaram como utilização nula no nmCRPC, utilização rara durante as mCRPC $1 \mathrm{~L}$ e $2 \mathrm{~L}$ e novamente com utilização nula pelos pacientes em linhas posteriores, mCRPC $3 \mathrm{~L}$ e $4 \mathrm{~L}+$, uma vez que seriam mais apropriados cuidados paliativos para essas fases da doença, e não a internação em UTI.

Há aumento dos custos mensais com os exames de imagem e laboratoriais ao longo das linhas de tratamento. Por exemplo, a solicitação do exame PSA se inicia com frequência trimestral para o $\mathrm{nmCRPC}$, e os especialistas relatam ser mensal a partir do momento que o paciente apresenta metástases. Exames de cintilografia óssea e tomografia do tórax, abdome e pelve são realizados semestralmente do nmCRPC até o MCRPC 2L e, de acordo com os especialistas, passam a ser trimestrais a partir da mCRPC 3L. A ressonância de coluna, direcionada para a avaliação da existência de compressão medular por conta da metástase óssea, é

$R \$ 420.000,00 \mathrm{R} \$ 440.000,00 \mathrm{R} \$ 460.000,00 \mathrm{R} \$ 480.000,00 \mathrm{R} \$ 500.000,00 \mathrm{R} \$ 520.000,00 \mathrm{R} \$ 540.000,00$

Custo de tratamento com hormonioterapia antiandrogênica de 2a geração nmCRPC

Tempo em hormonioterapia antiandrogênica de $2^{\text {a }}$ geração nmCRPC Custo de tratamento com inibidor seletivo de biossíntese de androgênio

Progressão para MCRPC $3 \mathrm{~L}$

Progressão para MCRPC $2 \mathrm{~L}$

Progressão para mCRPC 4L+

Custo de tratamento com cabazitaxel

Custo de tratamento com ADT

Custo de tratamento com radium-22

Tempo em internação clínica mCRPC 4L+

Valor diário de internação em clínica médica

Tempo em quimioterapia mCRPC $3 \mathrm{~L}$

Tempo em internação clínica MCRPC 3L

Custo de tratamento com docetaxel

Tempo em internação clínica MCRPC 2L

Porcentagem de metástases

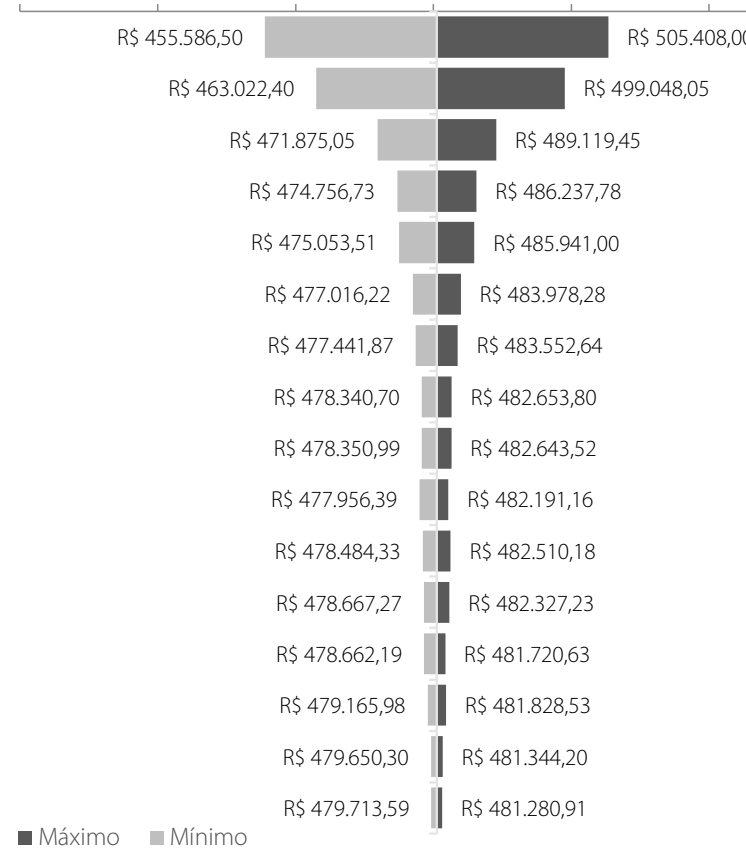

Figura 3. Análise de sensibilidade determinística. 
utilizada a partir da mCRPC 1L, com 10\% realizando o exame até um total de 100\% que realizam quando estão na mCRPC 4L+.

O uso de recursos gerais principalmente relacionados a cirurgias também foi potencialmente maior no quadro metastático. Por exemplo, nessa fase da doença, cerca de 90\% das metástases são ósseas e acarretam fraturas, compressão da medula, manejo da dor, entre outros. Nesse cenário, os custos das cirurgias relacionadas a fratura, descompressão medular e/ou manejo da dor com opioides aumentam ainda mais os custos do paciente com metástase. Os especialistas apontaram também para casos de metástase leptomeníngea. Nesse caso, alguns pacientes são elegíveis para a radioterapia, que possui um custo unitário relativamente alto (aproximadamente 16.000 BRL). Por outro lado, o paciente com nmCRPC também requer alguns procedimentos, como desobstrução prostática e duplo J, porém esses custos ainda são inferiores a todos os manejos de um paciente com metástase.

Os resultados desse estudo corroboram as conclusões de estudos que utilizaram outras metodologias, porém chegaram às mesmas conclusões em relação ao custo considerável de um paciente metastático. Li et al., em um estudo com dados de mundo real englobando 7.482 casos de pacientes com câncer de próstata com metástase e 25.709 pacientes sem metástase, avaliaram que os custos médicos são substancialmente maiores no cenário metastático (Li et al., 2017). Asano et al. utilizaram um banco de dados administrativo para avaliar os custos dos pacientes com câncer de próstata com metástase óssea no Sistema de Saúde Suplementar brasileiro. Da mesma forma, chegaram à conclusão de que os custos de um paciente com câncer de próstata metastático são elevados, e uma das principais causas é a maior quantidade de hospitalizações com maior duração de permanência (Asano et al., 2018). De fato, na mCRPC 4L+, os especialistas estimaram que $100 \%$ dos pacientes fariam uma internação clínica versus $1 \%$ no nmCRPC.

Embora nas primeiras fases da doença (nmCRPC) o tempo de tratamento e o número de pacientes sejam superiores em comparação aos estágios mais tardios de tratamento (mCRPC $3 \mathrm{~L}$ e $4 \mathrm{~L}$ ), provavelmente devido à progressão mais rápida da doença ou mortalidade, ainda assim o custo global do paciente mCRPC é superior ao do paciente nmCRPC. Além disso, mais do que uma questão de custos, a fase metastática está associada ao aparecimento de novos sintomas, que são mais agressivos e pioram a qualidade de vida do paciente, ocasionando impacto não só físico, mas também emocional. A progressão da doença é uma preocupação constante na vida do paciente que chega ao estágio metastático, uma vez que é muito rara a possibilidade de cura (NCCN, 2018; Eliasson et al., 2017; Herr \& O'Sullivan, 2000). Logo, são importantes tratamentos adequados para que se tente evitar ou prorrogar o tempo até a metástase, auxiliando não só do ponto de vista econômico, mas principalmente relativamente ao impacto social e físico no paciente.
As limitações desse estudo foram principalmente aquelas relacionadas à metodologia de um painel Delphi. Entre essas, a possibilidade de ocorrer um falso consenso, no qual os especialistas podem em alguns momentos apenas seguir a opinião de um dos membros. Além disso, o método tem que lidar com vieses de memória, como tendência de lembrar dos casos mais graves e dificuldade em lembrar de pacientes com menor número de complicações. Além disso, a formação do painel com especialistas na área de oncologia levou à dificuldade de obtenção de dados que não fazem parte da rotina de atendimento deles, por exemplo, os dados relacionados à utilização do pronto-socorro.

A referência dos custos foi retirada da CBHPM 2016, Simpro 2018 e CMED, mas os custos podem estar subestimados ou superestimados, já que variam dependendo de cada estabelecimento. Recursos como o PET-CT PSMA não foram englobados, pois os especialistas consideraram que não são reembolsados pelo Sistema de Saúde Suplementar, e sim out-of-pocket. Além disso, custos indiretos não foram incluídos, logo o custo do tratamento do câncer de próstata nesse aspecto possivelmente está subestimado.

\section{Conclusão}

Os custos do tratamento com medicamentos, exames, recursos hospitalares do câncer de próstata metastático são substancialmente superiores aos custos do tratamento do paciente não metastático resistente à castração de alto risco. Portanto, medidas que previnam ou adiem o surgimento de metástases podem ser economicamente importantes, além de ser fundamental diminuir os impactos da doença metastática no paciente.

\section{Agradecimentos}

A Simony Dantas, pelo trabalho de identificação de materiais necessários e custos associados aos recursos na Saúde Suplementar, e a Renata Eiras, pela moderação da fase presencial do painel Delphi.

\section{Referências bibliográficas}

Asano EW, Kim HSJ, del Aguila MC, Navarro S, Fernandes RA. Câncer de próstata com metástase óssea: impacto econômico para o Sistema de Saúde Suplementar brasileiro. J Bras Econ Saúde. 2018;10 (2):157-64.

AUA - American Urological Association. Castration-Resistant Prostate Cancer. 2018. Available from: https://www.auanet.org/guidelines/prostate-cancercastration-resistant-guideline. Accessed on: Jun 17, 2019.

AMB - Associação Médica Brasileira. Classificação Brasileira Hierarquizada de Procedimentos Médicos - CBHPM. Barueri, SP: Manole; 2016. Available from: https://amb.org.br/cbhpm/. Accessed on: Jun 17, 2019.

Brasil. Ministério da Saúde. Diretrizes Diagnósticas e Terapêuticas do Adenocarcinoma de Próstata: Comissão Nacional de Incorporação de Tecnologias no SUS. Brasília, DF: Ministério da Saúde; 2015. 
Brasil. Ministério da Saúde. Secretaria de Ciência, Tecnologia e Insumos Estratégicos. Departamento de Ciência e Tecnologia. Diretrizes metodológicas: análise de impacto orçamentário. Manual para o Sistema de Saúde do Brasil. Brasília, DF: Ministério da Saúde; 2014.

Eliasson L, de Freitas HM, Dearden L, Calimlim B, Lloyd AJ. Patients' Preferences for the Treatment of Metastatic Castrate-resistant Prostate Cancer: A Discrete Choice Experiment. Clin Ther. 2017;39(4):723-37.

Etges APBS, Schlatter RP, Neyeloff JL, Araújo DV, Bahia L, Cruz L, et al. Estudos de Microcusteio aplicados a avaliações econômicas em saúde: uma proposta metodológica para o Brasil. J Bras Econ Saúde. 2019;11(1):87-95.

Ferreira U, Netto Jr NR, Koff W, Pompeo AC, Sadi MV, Bretas FF, et al. Treatment of metastatic prostate cancer. 3rd Meeting of the Brazilian Committee of Uro-oncology Studies (COBEU). Curitiba, Brazil; 2004.

Gordon TJ. The Delphi method. Futures Research Methodology. 1994;2(3):1-30.

Heidenreich A, Bastian PJ, Bellmunt J, Bolla M, Joniau S, van der Kwast T, et al. EAU guidelines on prostate cancer. Part II: Treatment of advanced, relapsing, and castration-resistant prostate cancer. Eur Urol. 2014;65(2):467-79.

Herr HW, O'Sullivan M. Quality of life of asymptomatic men with nonmetastatic prostate cancer on androgen deprivation therapy. J Urol. 2000;163(6): 743-6.

Inca - Instituto Nacional de Câncer. Atlas de Mortalidade por Câncer. 2015. Available from: https://mortalidade.inca.gov.br/MortalidadeWeb/. Accessed on: Jul 10, 2019.
Inca - Instituto Nacional de Câncer. Tipos de câncer. Câncer de próstata. 2018. Available from: https://www.inca.gov.br/tipos-de-cancer/cancer-deprostata. Accessed on: Jul 10, 2019.

Li TT, Shore ND, Mehra M, Todd MB, Saadi R, Leblay G, et al. Impact of subsequent metastases on costs and medical resource use for prostate cancer patients initially diagnosed with localized disease. Cancer. 2017;123(18):3591-601.

Marques JBV, Freitas D. Método DELPHI: caracterização e potencialidades na pesquisa em Educação. Pro-Posições. 2018;29(2):389-415. Available from: http://www.scielo.br/scielo.php?script=sci_arttext\&pid=S010373072018000200389\&nrm=iso. Accessed on: Jun 17, 2019.

NCCN - National Comprehensive Cancer Network. NCCN Clinical Practice Guidelines in Oncology. (NCCN Guideline ${ }^{\oplus}$ ) Prostate Cancer. Version 4.2018. 2018. Available from: https://www.nccn.org. Accessed on: Jul 17, 2019.

Sasse AD, Reis RB, Nogueira LM, Maluf FC, Herchenhorn D, Smaletz O, et al. Second Brazilian consensus on the treatment of advanced prostate cancer: a SBOC-SBU-SBRT panel review. Int Braz J Urol. 2019;45(3):449-58.

SBOC - Sociedade Brasileira de Oncologia Clínica. Recomendações para o Tratamento do Câncer de Próstata. 2017.

Thangaratinam S, Redman CWE. The Delphi technique. Obstet Gynaecol. 2005;7:120-5. 